\title{
An Enhanced Energy Efficient Protocol with Optimize Cluster Head Selection Approach using Firefly Algorithm
}

\author{
Pankaj Soni $^{1}$, Rajnish Kansal ${ }^{2}$ \\ Dept of Computer Science \& Engg., ASRA Institute of Engineering and Technology,Bhawanigarh ${ }^{1}$ \\ Assistant Prof, Dept of Computer Science \& Engg,, ASRA Institute of Engineering and Technology,Bhawanigarh ${ }^{2}$
}

\begin{abstract}
Wireless Sensor Network is the combination of small sensor nodes which sense the data from its surroundings such as temperature etc. then the collected or sensed data travels from source node to sink node via a selected and efficient route. The process of data transmission accomplishes by consuming the amount of energy. The energy consumption directly affects the lifetime of the network. Hence the performance and lifetime of the network is depends upon the amount of energy consumed by the nodes. There are various protocols which are used for reducing the energy consumption of the nodes by various means such as selection or route to the sink node and section of cluster heads. So this paper provides a review to the various techniques used for selecting the cluster heads for the network. The cluster head selection is a crucial part of the network. Because the node selected as cluster head is responsible for data transmission between sources and sink node. So the candidate node for cluster head should have required amount of energy so that it can remain live for long period and enhances the performance and lifetime of the network.
\end{abstract}

Keywords: WSN, Low Energy Adaptive Clustering Hierarchy, Routing, Cluster heads, TEEN.

\section{INTRODUCTION}

The process of finding the data transmission route is termed as routing. In wireless sensor network large amount of nodes are present that are used for the sensing, transmitting, receiving of the data between the nodes present in the network. The route selected for the data transmission between source and sink node affects the lifetime or performance of the network. Because the energy consumed by the nodes while data transmission will be less if the selected route is small and energy consumption will be high if the route is long. The network efficiency depends on the energy consumed for transmitting the data by the nodes. Uses of WSN are increasing day by day without any kind of limitations. Different type of applications have different type of network bearing constraints and features but still most of the issues are common or same which makes them comparable. The positions of the sensor node become most sensitive point while the process of deployment of nodes. But sometimes coverage area of networks creates an issue because it also directly depends upon the positions of the nodes. In sniper systems, a sensor network is placed to secure an area from the snipers. The network is designed according to the urban areas where snipers can easily hide and sound sensors are also installed in the network to detect the sound generated by the bullet. By fetching data collected by all these sensors, the location of the sniper can be estimated after applying space temporal data in an efficient manner. In some cases complete terrain coverage is must where the situations are critical so that the sensors can detect the bullet trajectory no matter what this trajectory is. But in some areas like biomedical sensors the coverage of the terrain is not important but in this situation interface with the patient or the system's safety become of a critical. There are two other important Concern, In the first the WSN is deployed in a battlefield where the support and maintenance of WSN is not possible or a longer period of lifetime.

\section{TECHNIQUES}

Various clustering techniques for energy efficient WSN is as follows:
- LEACH
- HEED
- TEEN
- DEEC
- PEGASIS
- SEP

\section{LEACH}

LEACH means Low Energy Adaptive Clustering Hierarchy. It is a clustering protocol. It distributes the energy load equally among all the sensors in a network. Leach is a self-organized and adaptive protocol for networks. In LEACH, all the nodes contained in a local cluster and a single node among all of the nodes behaves like a cluster-head or base station. If the cluster heads were chosen a fixed throughout the system life time, as in conventional clustering algorithms, it is easy to see that 
the sensors selected for cluster-heads would die quickly, ending the useful lifetime of all nodes belonging to those clusters.

Therefore LEACH has a high energy cluster which is random so that it can move or rotate among all sensor nodes to drain the battery of single sensor. Due to the advantages of LEACH such as reduced control messages, bandwidth reusability, enhanced resource allocation, improved power control and lest wastage of energy it is proved efficient for Autonomic Sensor Network which has mobile battery power nodes.

\section{Advantages}

1. Easy to understand hence widely used in WSN as hierarchical routing.

2. Equal Load sharing. Because once a node is selected as Cluster head can't be a cluster head again in another round.

3. Due to the average energy consumption it is helpful to enhance the lifetime of the network.

Disadvantages

1. It affects the network robustness due to its feature of random selection of clusters heads. Because in this the chances of a node to become a cluster head is more or equal in every round irrespective to its remaining energy. Hence if the node with the lowest energy is selected as the cluster head then it can effect or reduce the lifetime of the network. Because the node with the minimum amount of energy will die quickly as compare to other nodes.

2. The randomization of cluster head leads to the unequal distribution which increases energy consumption and leave a negative impact on overall performance of the network.

3. In his scenario the cluster heads directly communicate to the sink node. It means the cluster heads which are located at the distance from the sink node also have to communicate directly to the sink node which results in the more energy consumption. Hence the far located cluster heads will crash earlier which decreases the performance of the network.

\section{HEED}

HEED stands for Hybrid Energy-Efficient Distributed Clustering. HEED does not contain any communication overhead so it did not facilitate the efficient distribution of cluster head nodes over the network. LEACH-C protocol is use to overcome this problem, it is a centralized approach but scalable to limited number of sensors only. Many clustering algorithms are available which creates more uniform clusters at the expense of overhead in cluster formation. One of the approaches is HEED which uses distributed algorithms which are easy and quick to convert. HEED uses a cluster formation algorithm, in which each node is assigned with a cluster head probability which is the function of their residual energy and also communication cost which is the function of neighbor proximity. Cluster head probability is used to decide that whether the sensor node is candidate for cluster head for this round or not.

Advantages

The advantage of HEED is that

1. There is no need to have the global knowledge regarding the nodes in a network

2. It terminates the processing at $\mathrm{O}$ (1) round or iteration,

3. It considers that each node is part of a single cluster heads.

Disadvantages

1. Generate more cluster heads, which are irrelevant or have no need to generate these cluster heads. Generation of irrelevant cluster heads leads to the imbalance in the level of energy consumption.

2. Due to the dissipation of energy the performance of the network gets affected.

3. In order to create cluster heads HEED requires large number of iterations and during each and every iteration lot of packets are broadcasted.

4. The cluster heads located near to the sink node exhausted earlier because it has huge work load.

\section{TEEN}

TEEN stands for Threshold sensitive Energy Efficient sensor Network protocol. It is used for reactive networks. It is an application which senses the temperature. It is more energy efficient protocol as compare to the conventional protocols. To overcome the limitations of LEACH protocol the TEEN protocol is developed. It enhanced version of LEACH protocol. TEEN is not suitable for large scale networks because it lacks:

- Randomly choosing cluster-heads before the events happened. It makes the sensors out of the event region gathering into clusters and transmitting data, causing unnecessary energy consumption and unbalance clusters.

- Choosing the cluster-heads without considering their residual energy. It may choose the sensors with less energy as the cluster-heads and then cause them premature death.

- The cluster-heads transmit data to the sink node directly. Thus one-hop transmission mode may result in the cluster-heads far away from the sink node quickly dead.

\section{Advantages}

1. It reduces the amount of energy used for transmission of the data by transmitting the necessary or sensitive data only.

2. It results in improvement in received data and efficiency of the network.

3. It has the feature of robustness.

Disadvantages

1. Because it is a threshold sensitive protocol the whole process relies on the threshold therefore this protocol is not suitable for periodic report applications. 
2. In TEEN the information is transmitted only at the Advantages cluster heads so in case if it is not able to find t5he cluster heads then the data is destroyed.

\section{DEEC}

DEEC stands for distributed energy efficient clustering. It is used for heterogeneous wireless sensor network. In this protocol the ratio between the remaining energy on each node and average energy on the network is calculated and its probability ratio is used to select the cluster-heads. The number of rotation on each node varies along with the variations in initial and remaining energy i.e. DEEC transforms the rotation on each node into energy. The nodes which have high amount of residual energy and high initial energy is much suitable for cluster head candidate node as compare to the nodes with low energy. Therefore DEEC can extend the lifetime of the network by using heterogeneous aware clustering algorithms. DEEC can gain more effective messages as compare to the classical clustering algorithms. DEEC is more suitable for Multilevel heterogeneous networks.

\section{Advantages}

Following are the advantages of DEEC:

1. Unlike other protocol there is no need of having the information of energy of rest of the nodes in each and every round.

2. DEEC is most suitable protocol for multi-level heterogeneous wireless networks.

\section{Disadvantages}

1. Advance nodes are rebuke in DEEC. Because when the energy of the advance nodes get exhausted then these fall under the category of normal nodes. Hence the advance nodes die rapidly as compare to other nodes in the network.

\section{PEGASIS}

PEGASIS stands for Power-Efficient Gathering in Sensor Information Systems. It is a chain-based power effective algorithm. It is based on following two parameters:

- Chaining

- Data fusion

It works similarly LEACH. In PEGASIS, each node can act as a leader of the chain and chain is also constructed with the help of greedy algorithm and can install by the sensor nodes. PEGASIS is based on following assumptions:

- Sensor nodes have global knowledge of the network

- All the nodes are stationary

- Nodes have knowledge about the location of all other nodes.

PEGASIS also face some problems as LEACH suffers. One of the main disadvantages of PEGASIS is that it is not scalable so it cannot be used in case of wireless sensor networks due to the lack of knowledge regarding the nodes because of large number of nodes.
1. PEGASIS is an enhanced version of LEACH protocol.

2. In this protocol the energy is distributed equally to all the sensor nodes so that the nodes can remain in working condition for long period.

\section{Disadvantages}

The disadvantage of the PEGASIS is :

1. The lifetime of the sensor node is small.

2. Because the nodes have to directly communicate the sink node which leads to the highest energy consumption for the nodes which are located far away from the sink node.

\section{SEP}

SEP is a protocol which supports the diversity in two levels of network. Here diversity refers to the initial energy allotment to the sensor nodes. According to the assumptions of SEP protocol in real time network there are two types of energy and hence it defines the nodes as advance nodes and normal nodes. The only difference between advance nodes and normal nodes is that the advance nodes have more amount of energy as compares to the normal nodes. On the basis of initial energy it assigns the weighted probability to nodes. It also overcomes the problem of cluster formation which exists in $\mathrm{LEACH}$ protocol.

\section{ADVANTAGES}

1. The advantage of SEP is that it doesn't require the information regarding the energy level of nodes at each election round.

\section{Disadvantages}

1. The disadvantage of the SEP is that in this there are two types of nodes in the network which leads to the conflicts while cluster head selection. In this cluster head selection is not dynamic. Hence resultant the normal nodes will exhaust first.

\section{RELATED WORK}

i. Supriya Dhauta et al [1]In this author describes that various clustering techniques are used in WSN and many surveys and research are conducted regarding this topic. WSN is a sensor network which runs on the basis of battery life i.e. nodes in this system operates through battery, in this way lifetime of the network does effected because nodes consumes more power. In earlier homogeneous systems the nodes are allotted with the equal amount of energy so that the lifetime of the system can be enhanced. But in case of heterogeneous networks different amount of energy is allotted to the nodes to increase the lifetime of the system. In this author defines clustering algorithm for both homogeneous and heterogeneous networks.

ii. Leena Y.Bara et al [2] In this author defines the LEACH protocol to increase the efficiency of the system. 
LEACH is an energy efficient protocol which is used to enhance the lifetime of the network. In LEACH nodes are categorized into clusters and cluster consist of related nodes only. Then from clusters a cluster head is selected which is used to transfer the data from clusters to Sink node. The communication link between cluster heads and sink nodes will be aborted when cluster head dies due to the insufficiency of energy or when communication is completed. Various parameters like PDR i.e. Packet Delivery Ratio, Delay or lifetime etc are used for evaluating the performance of the system. After evaluation it is observed that the proposed technique is much better than existing techniques.

iii. Harneet Kour et al [3]In this author represents the HEED for increasing the lifetime of the system along with increased efficiency of the system. HEED is a Hybrid Energy Efficient Distributed Protocol. It is a protocol which enhances the lifetime or efficiency of the system. The efficiency of the protocol is proved after getting the results which are much reliable as com[pare to traditional techniques.

iv. Sunita Rani, Er.Tarun Gulati, In this author defines that Wireless sensor network is an ad hoc network. In WSN each senor node has limited amount of energy to consume each sensor is defined with limited energy. These wireless sensors sense and monitor the data from its surroundings physical or environmental condition such as temperature etc. then the sensed data is stored and then transferred to the sink nodes. The process of data transmission consumes the amount of energy which directly affects the lifetime of the network. The protocols are used to minimize the delay in data transmission along with the reduced power consumption and extended lifetime of the network. Example is PEGASIS. PEGASIS follows a chain structure, every chain consist of only one cluster head, it is used corresponding to every node's receiving and sending messages who belong to this chain, the cluster head consumes more energy and the times of every round increasing. In PEGASIS, saves the energy for WSN and increase the lifespan of the network. In proposed work author proves that to select the next neighboring node is much reliable. For considers the some parameters like Distance, Residual Energy and Response time. As result simulates that PEGASIS leads to the reduction in energy consumption and extended lifetime of the network.

\section{v. Georgios Smaragdakis Ibrahim Matta Azer} Bestavros, In this paper the author explains the effect of heterogeneity of hubs, as far as their vitality, in remote sensor arranges that are progressively bunched. In these systems a portion of the hubs get to be group heads. The likewise accept that the sensors are arbitrarily (consistently) conveyed and are not portable, the directions of the sink and the measurements of the sensor field are known. The author demonstrates that the conduct of such sensor systems turns out to be exceptionally flimsy once the primary hub passes on, particularly in the nearness of hub heterogeneity. Established bunching conventions accept that all the nodes are outfitted with the same measure of vitality and thus, they cannot exploit the nearness of hub heterogeneity. The author propose SEP, a heterogeneous-mindful convention to delay the time interim before the passing of the main hub (we allude to as security period), which is essential for some applications where the input from the sensor system must be dependable. SEP depends on weighted race probabilities of every hub to wind up cluster head as indicated by the remaining vitality in every hub. The author appears by recreation that SEP dependably drags out the dependability period contrasted with (and that the normal throughput is more prominent than) the one got utilizing current grouping conventions. The author closes by considering the affectability of our SEP convention to heterogeneity parameters catching vitality awkwardness in the system. The author found that SEP yields longer security locale for higher estimations of additional vitality brought by all the more capable hubs.

\section{CONCLUSION AND FUTURE SCOPE}

After reading the related work section it is concluded that the wireless sensor network relies on the amount of energy consumed by its nodes for data transmission and the nodes which are selected as the cluster heads directly affects the performance of the network. There a4re various techniques have been developed which are helpful for efficient cluster head selection and route selection. But all of these techniques have some lacking point.

So in future there is a need to develop such a system which can increase the efficiency of the network and also enhances the lifetime of the network.

\section{REFERENCES}

[1] Supriya Dhauta,"Review on LEACH- Homogeneous and heterogenous Wireless sensor network", ijircce, vol 3(5), Pp 44424448, 2015

[2] Leena y. bara, "Performance evaluation of LEACH protocol for WSN", ijirae, vol 1(6), Pp 141-145, 2014

[3] Harneet Kaur, "Hybrid energy efficient Dustributed protocol for heterogenous WSN", ijca, vol 4(6), Pp 1-5, 2010

[4] Sunita Rani, Er.Tarun Gulati, "an improved pegasis protocol to enhance energy utilization in wsn”, ijccr, vol 2 (3), 2012

[5] Georgios Smaragdakis Ibrahim Matta Azer Bestavros, "SEP: A Stable Election Protocol for clustered heterogeneous wireless sensor networks", Pp 1-11, 2013

[6] Yan Sun, "Energy-Efficient Routing Protocol in Event-Driven Wireless Sensor Networks", IEEE, May 2010, pp 1-5,

[7] Tripti Sharma,"Performnace comparison of LEACH, SEP and DEEC Protocol in wireless sensopr network" ,Pp 10-15

[8] Samayveer Singh, "Heterogeneous protocols for increasing the life time of wireless sensor networks", Journal of Global Research in Computer Science Vol. 2, No.4, April 2011, Pp.172-176

[9] S Karthikeyan,"Energy Efficient System for Heterogeneous wireless sensor networks", European Journal of Scientific Research Vol.7 2, No.4, 2012, Pp.599-607.

[10] Manju Bala, "On Proficiency of HEED protocol with Heterogeneity for Wireless Sensor Networks with BS and Nodes Mobility", International Journal of Applied Information Systems, Vol.2, No. 7, May 2012, Pp. 56-62. 
[11] Harneet Kour, Ajay K. Sharma "Hybrid Energy Efficient Distributed Protocol for Heterogeneous Wireless Sensor Network", International Journal of Computer Applications ,Vol.4, No.6,July 2010, Pp. 37- 41

[12] Q. Li, "Design of a distributed energy efficient clustering algorithm for heterogeneous wireless sensor networks". Computer Communications, vol. 29, 2006, Pp. 2230- 2237.

[13] Yingcghi Mao,"An Effective Data Gathering Scheme in Heterogeneous Energy Wireless Sensor Network". In International Conference on Computational Science and Engineering,2009, Pp. 338-343

[14] Arth Shaileshbhai Raval "Energy Efficient Cluster Head Selection for Data Aggregation in Wireless Sensor Networks" International Journal of Advanced Research in Computer Science and Software Engineering, Volume 4, Issue 2, February 2014 ,Pp 128-131

[15] Asaduzzaman, "Energy efficient cooperative LEACH protocol for wireless sensor networks: Communications and Networks, Journal of ,Volume:12, Issue: 4, 20 December, 2012, Pp 358-365

[16] Chunyao FU "An Energy Balanced Algorithm of LEACH Protocol in WSN "IJCSI International Journal of Computer Science Issues, Vol. 10, Issue 1, No 1, 2013, Pp 354-359

[17] Dr.R.KalaiMagal "A SURVEY ON WIRELESS SENSOR NETWORK PROTOCOLS", ISET - International Journal of Innovative Science, Engineering \& Technology, Vol. 1 Issue 6, 2014, Pp 548-562

[18] EunHwa Kim "Cluster Head Selection Based On Hop Count in a Wireless Sensor Networks" Advanced Science and Technology Letters , 2014, Vol.51, 2014, Pp.124-127

[19] Harneet Kour," Hybrid.Energy Efficient Distributed Protocol for Heterogeneous Wireless Sensor Network International Journal of Computer Applications, Volume 4 - No.6, 2010, Pp 1-5.

[20] Hemavathi Natarajan "A Fuzzy Based Predictive Cluster Head Selection Scheme for Wireless Sensor Networks" 4 September,2014.

[21] Jay G. Shah "Technical Review of Wireless Sensor Network and its Protocol "International journal for research in emerging science and technology, volume-2, issue-1, 2015, Pp 35-41.

[22] Jenn-Long Liu "LEACH-GA: Genetic Algorithm-Based EnergyEfficient Adaptive Clustering Protocol for Wireless Sensor Networks, . IJMLC 2011 Vol.1(1), 1 April,2013, Pp 79-85 .

[23] Jong-Shin Chen "Efficient Cluster Head Selection Methods for Wireless Sensor Networks" Journal of networks, VOL. 5, NO. 8, AUGUST 2010, Pp 964-970

[24] Jong-Yong Lee "Improving the Energy Efficiency of a Cluster Head Election for Wireless Sensor Networks", 25 March,2014.

[25] K.Gandhimathi "A Survey on Energy Efficient Routing Protocol in Wireless Sensor Network" , International Journal of Advance Research Computer Science and Management Studies, Vol 2,issue 8, 2014, Pp 174-181

[26] V.Preetha "Clustering \& Cluster Head Selection Techniques in Mobile Adhoc Networks" International Journal of Innovative Research in Computer and Communication Engineering, Vol. 2, Issue 7, July 2014, Pp 5151-5157

[27] Ying Liang "Energy Adaptive Cluster-Head Selection for Wireless Sensor Networks" IEEE, December,2005, Pp 634 - 638

[28] Shital Y. Agrawal "A Survey on Location Based Routing Protocols for Wireless Sensor Network "International Journal of Emerging Technology and Advanced Engineering Website, Volume 3, Issue 9, 2013, Pp 123-126

[29] Rajesh chaudhary,"Review paper on energy efficient protocols in wireless sensor networks', iosrjen, vol4(2), Pp 2278-8719, 2014

[30] Koen Langendoen,"Distributed localization in wireless sensor networks: a quantitative comparison ",ELSEVIER, 2003,Pp 499518 